\title{
Crystal structure of 2-isopropylthioxanthone, $\mathrm{C}_{16} \mathrm{H}_{14} \mathrm{OS}$
}

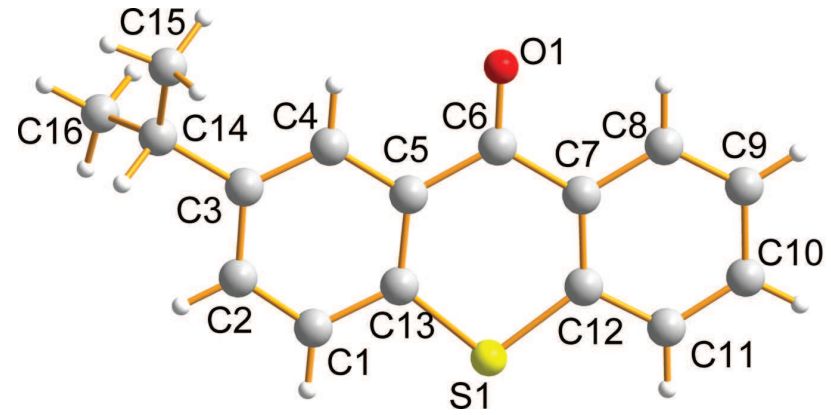

https://doi.org/10.1515/ncrs-2018-0576

Received December 10, 2018; accepted January 15, 2019; available online January 31, 2019

\section{Abstract \\ $\mathrm{C}_{16} \mathrm{H}_{14} \mathrm{OS}$, monoclinic, $P 2_{1} \quad$ (no. 4), $a=8.1588$ (5) $\AA$, $b=7.8670(4) \AA, \quad c=10.2161(5) \AA, \quad V=655.70(6) \AA^{3}, \quad Z=2$, $R_{\mathrm{gt}}(F)=0.0539, w R_{\mathrm{ref}}\left(F^{2}\right)=0.1312, T=290 \mathrm{~K}$.}

\section{CCDC no.: 1890930}

The crystal structure is shown in the figure. Tables 1 and 2 contain details on crystal structure and measurement conditions and a list of the atoms including atomic coordinates and displacement parameters.

\section{Source of material}

The title compound was purchased from Sinopharm Chemical Reagent Co. Ltd. (Shanghai). Light yellow crystals suitable for X-ray diffraction study were obtained from a saturated toluene solution with cooling crystallization.

\section{Experimental details}

All of the non-hydrogen atoms were refined anisotropically. The hydrogen atoms were assigned with common isotropic

*Corresponding author: Jin-Qiang Liu, College of Chemistry and Chemical Engineering, Luoyang Normal University, Luoyang, Henan 471022, P. R. China, e-mail: liuque.zju@hotmail.com

Li Ji: Zhejiang Medicine Co., Ltd., R\&D Center, Xinchang, Zhejiang Province 312500, P. R. China Luoyang Normal University, Luoyang, Henan 471022, P. R. China
Weizhou Wang: College of Chemistry and Chemical Engineering,

Table 1: Data collection and handling.

\begin{tabular}{ll}
\hline Crystal: & Yellow block \\
Size: & $0.27 \times 0.26 \times 0.25 \mathrm{~mm}$ \\
Wavelength: & Mo $K \alpha$ radiation $(0.71073 \AA)$ \\
$\mu:$ & $0.23 \mathrm{~mm}^{-1}$ \\
Diffractometer, scan mode: & SuperNova, $\omega$ \\
$\theta_{\text {max }}$, completeness: & $29.3^{\circ},>99 \%$ \\
$N(h k l)_{\text {measured }}, N(h k l)_{\text {unique }}, R_{\text {int }}:$ & $5364,2923,0.022$ \\
Criterion for $I_{\text {obs }}, N\left(h k l_{\text {gt }}:\right.$ & $I_{\text {obs }}>2 \sigma\left(I_{\text {obs }}\right), 2582$ \\
$N(\text { param })_{\text {refined }}:$ & 165 \\
Programs: & CrysAlis $^{\mathrm{PRO}}[1]$, SHELX $[2,3]$ \\
\hline
\end{tabular}

Table 2: Fractional atomic coordinates and isotropic or equivalent isotropic displacement parameters $\left(\AA^{2}\right)$.

\begin{tabular}{lrrrr}
\hline Atom & $\boldsymbol{x}$ & $\boldsymbol{y}$ & $\boldsymbol{z}$ & $\boldsymbol{U}_{\text {iso }}{ }^{*} \boldsymbol{U}_{\text {eq }}$ \\
\hline S1 & $0.50169(13)$ & $0.3258(2)$ & $0.50717(10)$ & $0.0574(3)$ \\
O1 & $0.1820(4)$ & $0.5895(6)$ & $0.2193(3)$ & $0.0821(11)$ \\
C1 & $0.7057(5)$ & $0.3465(6)$ & $0.3068(4)$ & $0.0575(10)$ \\
H1 & 0.7759 & 0.2866 & 0.3622 & $0.069^{*}$ \\
C2 & $0.7561(5)$ & $0.3928(7)$ & $0.1847(4)$ & $0.0696(13)$ \\
H2 & 0.8622 & 0.3665 & 0.1591 & $0.084^{*}$ \\
C3 & $0.6543(5)$ & $0.4773(7)$ & $0.0986(4)$ & $0.0639(12)$ \\
C4 & $0.4980(5)$ & $0.5176(5)$ & $0.1393(4)$ & $0.0542(10)$ \\
H4 & 0.4281 & 0.5747 & 0.0819 & $0.065^{*}$ \\
C5 & $0.4414(5)$ & $0.4752(5)$ & $0.2646(3)$ & $0.0451(8)$ \\
C6 & $0.2708(5)$ & $0.5198(5)$ & $0.2990(4)$ & $0.0510(9)$ \\
C7 & $0.2099(4)$ & $0.4795(5)$ & $0.4315(3)$ & $0.0447(8)$ \\
C8 & $0.0491(5)$ & $0.5289(5)$ & $0.4617(4)$ & $0.0582(10)$ \\
H8 & -0.0150 & 0.5829 & 0.3985 & $0.070^{*}$ \\
C9 & $-0.0145(6)$ & $0.4986(6)$ & $0.5835(5)$ & $0.0736(14)$ \\
H9 & -0.1209 & 0.5327 & 0.6021 & $0.088^{*}$ \\
C10 & $0.0790(6)$ & $0.4172(7)$ & $0.6790(5)$ & $0.0708(13)$ \\
H10 & 0.0356 & 0.3982 & 0.7615 & $0.085^{*}$ \\
C11 & $0.2351(5)$ & $0.3651(5)$ & $0.6515(4)$ & $0.0582(11)$ \\
H11 & 0.2969 & 0.3088 & 0.7149 & $0.070^{*}$ \\
C12 & $0.3019(5)$ & $0.3966(5)$ & $0.5278(3)$ & $0.0452(8)$ \\
C13 & $0.5477(5)$ & $0.3898(5)$ & $0.3482(3)$ & $0.0459(8)$ \\
C14 & $0.7167(7)$ & $0.5189(9)$ & $-0.0377(4)$ & $0.0897(19)$ \\
H14 & 0.7875 & 0.4221 & -0.0583 & $0.108^{*}$ \\
C15 & $0.5995(8)$ & $0.5186(9)$ & $-0.1415(5)$ & $0.100(2)$ \\
H15A & 0.5429 & 0.4116 & -0.1430 & $0.150^{*}$ \\
H15B & 0.6543 & 0.5352 & -0.2232 & $0.150^{*}$ \\
H15C & 0.5221 & 0.6088 & -0.1283 & $0.150^{*}$ \\
C16 & $0.8335(7)$ & $0.6665(9)$ & $-0.0331(5)$ & $0.0956(18)$ \\
H16A & 0.7768 & 0.7661 & -0.0034 & $0.143^{*}$ \\
H16B & 0.8760 & 0.6867 & -0.1191 & $0.143^{*}$ \\
H16C & 0.9223 & 0.6411 & 0.0260 & $0.143^{*}$ \\
\hline & & & &
\end{tabular}

๖ Open Access. @ 2019 jin-Qiang Liu et al., published by De Gruyter. (c) BY This work is licensed under the Creative Commons Attribution 4.0 Public License. 
displacement factors $U_{\text {iso }}(\mathrm{H})=1.2$ times $U_{\text {eq }}(\mathrm{C}$, aromatic ring and methenyl), and $U_{\text {iso }}(\mathrm{H})=1.5$ times $U_{\text {eq }}(\mathrm{C}$, methyl). The final refinement by using geometrical restraints, with $\mathrm{C}-$ $\mathrm{H}=0.93 \AA$ (aromatic ring), $\mathrm{C}-\mathrm{H}=0.98 \AA$ (methenyl), and C$\mathrm{H}=0.96 \AA$ (methyl). The Flack parameter $\mathrm{x}=0.04$ (5) [4] was determined using 903 quotients $[(\mathrm{I}+)-(\mathrm{I}-)] /[(\mathrm{I}+)+(\mathrm{I}-)]$.

\section{Comment}

2-Isopropylthioxanthone (ITX), has been widely used in many processes such as printing inks, surface coatings, microelectronics, and photoresists [5]. Nevertheless, it was also widely used in UV curing to promote the polymerization of inks used in paper- or plastic-based packaging materials because of its high photoinitiation efficiency and absorption characteristics at near-UV range $(370-385 \mathrm{~nm}$ ) [6-9]. To obtain the basic experimental data of ITX, we had measured the solubility of ITX in seven different pure solvents from 299.15 to $329.85 \mathrm{~K}[10]$, which was very important for its large scale synthesis with high purity. When we completed that work, we found that the crystal structure of the title compound was not reported up to now.

A view of the molecular structure of the title compound is given in the Figure. The asymmetric unit contains one molecule of the title compound, which is constructed by the thioxanthone, and the isopropyl fragment. The whole thioxanthone is almost planar with the maximum deviation of $0.042 \AA$ for $\mathrm{C} 10$. The bond lengths of $\mathrm{C} 12-\mathrm{S} 1$ and $\mathrm{C} 13-\mathrm{S} 1$ are 1.737(4) and 1.745(4) $\AA$, respectively. The values are intermediate between the double and single bonds. The shortening of the $\mathrm{C}-\mathrm{S}$ bonds showing the partial double-bond character. Bond lengths and angles are in accord with related structure [11].
Acknowledgements: This work was supported by the Key Scientific Research Foundation of Education Committee of Henan province (No. 17B530003).

\section{References}

1. Agilent Technologies: CrysAlis ${ }^{\text {PRO }}$ Software system. Agilent Technologies UK Ltd, Oxford, UK (2015).

2. Sheldrick, G. M.: SHELXS97. Program for the solution of crystal structures. University of Göttingen, Germany (1997).

3. Sheldrick, G. M.: SHELXL97. Program for the refinement of crystal structures. University of Göttingen, Germany (1997).

4. Parsons, S.; Flack, H. D.; Wagner, T.: Use of intensity quotients and differences in absolute structure refinement. Acta Crystallogr. B69 (2013) 249-259.

5. Roffey, C.: Photogeneration of reactive species for UV-curing. Wiley, Sussex, UK (1997).

6. Corrales, T.; Peinado, C; Catalina, F.; Neumann, M. G.; Allen, N. S.; Rufs, A. M.; Encinas, M. V.: Photopolymerization of methyl methacrylate initiated by thioxanthone derivatives: photoinitiation mechanism. Polymer 41 (2000) 9103-9109.

7. Corrales, T.; Catalina, F.; Peinado, C.; Allen, N. S.; Rufs, A. M.; Bueno, C.; Encinas, M. V.: Photochemical study and photoinitiation activity of macroinitiators based on thioxanthone. Polymer 43 (2002) 4591-4597.

8. Aydin, M.; Arsu, N.; Yagci, Y.: One-component bimolecular photoinitiating systems. Macromol. Rapid Commun. 24 (2003) 718-723.

9. Valderas, C.; Bertolotti, S.; Previtali, C. M.; Encinas, M. V.: Influence of the amine structure on the polymerization of methyl methacrylate photoinitiated by aromatic ketone/amine. J. Polym. Sci., A: Polym. Chem. 40 (2002) 2888-2893.

10. Zhang, Y.; Wang, H. H.; Wei, S.; Liu, J. Q.; Wang, W. Z.: Determination and correlation of solubilities of 2 isopropylthioxanthone (ITX) in seven different solvents from (299.15 to 329.85) K. J. Chem. Eng. Data 60 (2015) 941-946.

11. Richardson, M. F.; Guzowski, R. S.: Structures of 2-, 3-, and 4methyl-9-oxothioxanthenes. J. Crystallogr. Spect. Research 23 (1993) 633-639. 